\title{
Study Day of Cardiovascular System Findings
}

National Cancer Institute

\section{Source}

National Cancer Institute. Study Day of Cardiovascular System Findings. NCI Thesaurus.

Code C123950.

The study day that a cardiovascular system assessment is performed. 